\title{
Major events in multimedia information retrieval
}

\author{
Erwin M. Bakker ${ }^{1}$
}

Received: 18 October 2016 / Accepted: 21 October 2016 / Published online: 2 November 2016

(C) Springer-Verlag London 2016

Multimedia information retrieval (MIR) is a relatively young field in computer science. There are several well-established scientific meetings which produce high-quality peer-reviewed contributions. This editorial summarizes the current major events and meetings.

The top conference in MIR is the ACM International Conference on Multimedia Retrieval (ICMR). The roots of the meeting began in 2002 with the Challenges in Image and Video Retrieval (CIVR) conferences and then combined with several other meetings in 2011 for the first ICMR. Within 3 years, ICMR had already achieved international recognition and was considered by some of the more influential conference rankings (e.g. CCF) to be a top conference in the wide domain of graphics and multimedia. Furthermore, Google conference rankings placed it in the top ten of all multimedia journal and conference publications in terms of citations. ICMR is sponsored by SIGMM and typically held in June:

"Effectively and efficiently retrieving information based on user needs is one of the most exciting areas in multimedia research. The Annual ACM International Conference on Multimedia Retrieval (ICMR) offers a great opportunity for exchanging leading-edge multimedia retrieval ideas among researchers, practitioners and other potential users of multimedia retrieval systems.

This annual conference, which puts together the longlasting experiences of the former ACM CIVR and ACM MIR series, was set up in 2011 to illuminate the state

Erwin M. Bakker

erwinmbakker@gmail.com

1 LIACS Media Lab, Leiden University, Leiden, The Netherlands of the arts in multimedia (text, image, video and audio) retrieval."

See http://www.acmicmr.org and http://www.icmr2016. $\mathrm{com} /$.

The International Workshop on Content-based Multimedia Indexing (CBMI) is a high-quality meeting typically held in Europe in June:

" CBMI aims at bringing together the various communities involved in all aspects of content-based multimedia indexing for retrieval, browsing, visualization and analytics.

In addition to multimedia and social media search and retrieval, we wish to highlight related and equally important issues that build on content-based indexing, such as multimedia content management, user interaction and visualization, and media analytics.

Additional special sessions are planned in areas such as deep learning, medical image retrieval, big data and eLearning."

See http://cbmi2016.upb.ro.

The International Conference on Multimedia is a longrunning conference since 1993. It is the top scientific multimedia meeting in the world and has an excellent reputation in all of the international rankings. It is typically held in October:

"In the past decade, there has been an explosive growth of multimedia contents on the Web, desktops, and mobile devices. The deluge of multimedia leads to "information overload" and poses new challenges and requirements for effective and efficient access to multimedia content. Multimedia search and 
recommendation techniques are essential to provide information relevant to users' information needs.

This area calls for contributions on reporting novel problems, solutions, models, and/or theories that tackle the key issues in searching, recommending, and discovering multimedia content, as well as a variety of multimedia applications based on search and recommendation technologies."

See http://www.acmmm.org.

The MediaEval Benchmark is a relatively recent and influential project which focuses on multimedia evaluation. The workshop where results are shared is typically held roughly in October.

"MediaEval is a benchmarking initiative dedicated to evaluating new algorithms for multimedia access and retrieval. It emphasizes the 'multi' in multimedia and focuses on human and social aspects of multimedia tasks. MediaEval attracts participants who are interested in multimodal approaches to multimedia involving speech recognition, multimedia content analysis, music and audio analysis, user-contributed information (tags, tweets), viewer affective response, social networks, temporal and geo-coordinates."
See http://www.multimediaeval.org/.

TRECVID is the top video retrieval event in the world and has had a deep influence for both researchers and practitioners. The precursor events began in 2001 and the first TRECVID was held in 2003.

"The goal of the conference series is to encourage research in information retrieval by providing a large test collection, uniform scoring procedures, and a forum for organizations interested in comparing their results. In 2001 and 2002, the TREC series sponsored a video "track" devoted to research in automatic segmentation, indexing, and content-based retrieval of digital video. Beginning in 2003, this track became an independent evaluation (TRECVID) with a workshop taking place just before TREC."

See http://trecvid.nist.gov/. 